\title{
Inter-individual variability and differential tissue abundance of mitochondrial amidoxime reducing component (mARC) in humans
}
Deepak Ahire ${ }^{1}$, Abdul Basit ${ }^{1}$, Lisa J. Christopher ${ }^{2}$, Ramaswamy Iyer ${ }^{2}$, J. Steven Leeder ${ }^{3}$ and Bhagwat Prasad ${ }^{1}$

${ }^{1}$ Department of Pharmaceutical Sciences, Washington State University, Spokane, WA

${ }^{2}$ Department of Nonclinical Disposition and Bioanalysis, Bristol Myers Squibb Princeton, NJ

${ }^{3}$ Department of Pediatrics, Children's Mercy Hospitals and Clinics, Kansas City, MO

Corresponding Author: Bhagwat Prasad, Ph.D.

Address: Department of Pharmaceutical Sciences, Washington State University, 412 E Spokane Falls Blvd. Spokane, WA 99202. Email: bhagwat.prasad@wsu.edu. Phone: 509-358-7739 
Running title: Protein abundance of mARC enzymes in human

\author{
Abbreviations: \\ ABC, ammonium bicarbonate; AO, aldehyde oxidase; BCA, bicinchoninic acid; BSA, bovine \\ serum albumin; CHIM, cryopreserved human intestinal mucosa; CL, clearance; CYB5B, \\ cytochrome b5; CYB5R, cytochrome b5 reductase; HSA, human serum albumin; IVIVE, in \\ vitro to in vivo extrapolation; $\mu \mathrm{LC}-\mathrm{MS} / \mathrm{MS}$, microflow-liquid chromatography-mass \\ spectrometry; NADH, nicotinamide adenine dinucleotide; PBPK, physiologically-based \\ pharmacokinetic modeling; SIL, stable-isotope labeled, SO, sulfite oxidases; XO, xanthine \\ oxidases
}

Number of words in
Abstract: 250
Introduction: 511
Discussion: 1102

Figures: 4

References: 27 


\begin{abstract}
Mitochondrial amidoxime-reducing component (mARC) enzymes are molybdenum-containing proteins that metabolize a number of endobiotics and xenobiotics. The interindividual variability and differential tissue abundance of $\mathrm{mARC} 1$ and $\mathrm{mARC} 2$ were quantified using targeted proteomics in three types of tissue fractions: i) pediatric liver tissue homogenates, ii) total membrane fraction of the paired liver and kidney samples from pediatric and adult donors, and iii) pooled S9 fractions of the liver, intestine, kidney, lung, and heart. The absolute levels of mARC1 and mARC2 in the pediatric liver homogenate were $40.08 \pm 4.26$ and $24.58 \pm 4.02$ $\mathrm{pmol} / \mathrm{mg}$ homogenate protein, respectively, and were independent of age and sex. In the total membrane fraction of the paired liver and kidney samples, the abundance of hepatic mARC1 and mARC2 was comparable, whereas mARC2 abundance in the kidney was 9-fold higher in comparison to mARC1. The analysis of the third set of samples (i.e., S9 fraction) revealed that mARC1 abundance in the kidney, intestine, and lung was 5 to 13 -fold lower than the liver S9 abundance, whereas mARC2 abundance was $~ 3$ - and 16-fold lower in the intestine and lung than the liver S9, respectively. In contrast, the kidney mARC2 abundance in the S9 fraction was $~ 2.5-$ fold higher as compared to the hepatic mARC2 abundance. The abundance of mARC enzymes in the heart was below the limit of quantification $(\sim 0.6 \mathrm{pmol} / \mathrm{mg}$ protein). The $\mathrm{mARC}$ enzyme abundance data presented here can be used to develop physiologically based pharmacokinetic models for the prediction of in vivo pharmacokinetics of mARC substrates.
\end{abstract}




\section{Significance statement}

A precise targeted quantitative proteomics method was developed and applied to quantify newly discovered drug-metabolizing enzymes, mitochondrial amidoxime-reducing component (mARC) in pediatric and adult tissue samples. The data suggest that mARC enzymes are ubiquitously expressed in an isoform-specific manner in the human liver, kidney, intestine, and lung, and the enzyme abundance is not associated with age and sex. These data are important for developing physiologically based pharmacokinetic models for the prediction of in vivo pharmacokinetics of mARC substrates. 


\section{INTRODUCTION}

Molybdenum (Mo) containing enzymes play important roles in human physiology by catalyzing a range of redox reactions of carbon, sulfur, and nitrogen-containing endo- and xenobiotics (Krompholz et al., 2012; Plitzko et al., 2013; Jakobs et al., 2014a; Plitzko et al., 2015; Llamas et al., 2017). Mo-containing enzymes belong to two main families, xanthine oxidases (XO) and sulfite oxidases ( $\mathrm{SO}$ ), which contain enzymes such as aldehyde oxidase (AO) and mitochondrial amidoxime reducing-component (mARC), respectively. XOs are well known for their roles in the metabolism of drugs and endobiotics, especially due to the emerging importance of AO in the biotransformation of new chemical entities (Garattini and Terao, 2012; Sanoh et al., 2015); however, mARC enzymes are not well characterized for their expression and activity (Schwarz et al., 2009).

mARC enzymes are localized in the outer mitochondrial membrane with the catalytic domain facing the cytosol. They need two partner proteins for activity, i.e., cytochrome b5 (CYB5B), and cytochrome b5 reductase (CYB5R) (Jakobs et al., 2014b) (Fig. 1A). The three-enzyme system, which utilizes nicotinamide adenine dinucleotide (NADH) as the co-factor, is responsible for the reduction of N-oxygenated compounds (Fig. 1B). The mARC enzyme category has two homologous isoforms ( 66\% protein similarity), mARC1 and mARC2 (337 and 335, amino acids, respectively). mARC1 and mARC2 are known to be involved in lipid metabolism, nitric oxide (NO) homeostasis, and detoxification of mutagenic N-hydroxylated nucleobases (Havemeyer et al., 2011; Krompholz et al., 2012; Jakobs et al., 2014a; Maia and Moura, 2015; Schneider et al., 2018). Further, mARC enzymes reduce a variety of N-oxygenated xenobiotic compounds (e.g., N-oxides, N-hydroxy compounds, and amidoxime prodrugs). For example, amidoxime prodrugs (e.g., ximelagatran) rely on mARC enzymes for bioactivation in 
the gut to address the poor bioavailability due to protonation of amidine-containing active moieties (e.g., melagatran). Further, human mARC enzymes are also implicated in the metabolism of N-hydroxy sulfonamide (e.g., cimlanod) (Cowart et al., 2019a), N-oxides (e.g., amitriptyline-N-oxide and nicotinamide-N-oxide), oximes (e.g., 2,4,6-trimethylacetophenone oxime), and N-hydroxyamidinohydrazones (e.g., guanoxabenz) (Fig. 1B). All these reports highlight the pivotal role of the mARC enzymes in human physiology and xenobiotic metabolism.

To predict the pharmacokinetics of mARC substrates, it is important that the protein abundance of mARC in different tissues and their interindividual variability data are available. However, little is currently known about the absolute abundance of mARC enzymes, their differential tissue abundance, and the association of abundance with age and sex in humans. The aim of this study was to develop and apply a microflow-liquid chromatography-mass spectrometry ( $\mu$ LCMS/MS) quantitative proteomic method for the quantification of mARC enzymes for the investigation of inter-individual variability and differential tissue abundance (liver, kidney, intestine, lung, and heart) of mARC1 and mARC2 in humans. Further, we applied the method to quantify mARC1 and mARC2 in human hepatocytes and cryopreserved human intestinal mucosa (CHIM). The data generated in this study can be used for the characterization of inter-tissue variability in mARC proteins, characterization of in vitro models and in vitro to in vivo extrapolation (IVIVE), and in the improvement of the physiologically-based pharmacokinetic modeling (PBPK) model predictions of in vivo pharmacokinetics of mARC substrates. 


\section{MATERIAL AND METHODS}

\section{Materials}

Ammonium bicarbonate (ABC, 98\% purity), bovine serum albumin (BSA), dithiothreitol (DTT), iodoacetamide (IAA), protease inhibitor cocktail, and trypsin (MS grade) were procured from Thermo Fisher Scientific (Rockford, IL). Mem-PER Plus membrane protein extraction kit, Pierce bicinchoninic acid (BCA) protein assay kit, Optima MS-grade acetonitrile, chloroform, methanol, and formic acid were procured from Fisher Scientific (Fair Lawn, NJ). Stable isotopelabeled (SIL) peptides of mARC1 (DLLPIK and VGDPVYLLGQ) and mARC2 (LSPLFGIYYSVE and WFTNFL) (Table S1) for the quantification of mARC enzymes, were purchased from Thermo Fisher Scientific (Rockford, IL). The recombinant mARC1 and mARC2 protein standards were purchased from OriGene (Rockville, MD). Human serum albumin was purchased from Calbiochem (Billerica, MA).

\section{Procurement of tissue samples}

Due to the limited availability of pediatric tissue samples, frozen tissue fractions available in our laboratory from three previous studies (Prasad et al., 2014; Li et al., 2019; Basit et al., 2020b) were utilized for the quantification of mARC enzymes. These samples were: i) pediatric liver homogenate samples $(\mathrm{n}=79$, male-68\%, female-32\%), ii) paired pediatric and adult liver and kidney samples ( $\mathrm{n}=17$, male-65\%, female-35\%), and iii) pooled $\mathrm{S} 9$ fractions of liver (male$60 \%$, female-40\%), intestine (male-67\%, female-33\%), kidney (male-67\%, female-33\%), lung

(73\%, female-27\%), and heart (male-59\%, female-41\%). The pediatric and adult liver and kidney samples were procured from the Eunice Kennedy Shriver National Institute of Child Health and Human Development Brain and Tissue Bank for Developmental Disorders at the 
University of Maryland. The sample details are provided in Supplementary Table S2 and elsewhere (Bhatt et al., 2019; Li et al., 2019). The pediatric liver samples $(n=79)$ were categorized into four age categories: infancy ( $<1$ year), early childhood ( 1 to $<6)$, middle childhood (6 to <12), and adolescence (12 to <18 years). The pooled S9 fraction samples were either purchased from commercial vendors or prepared in house, i.e., the liver and intestinal S9 samples were obtained from Xenotech (Kansas City, KS), lung S9 fractions were provided by Dr. Scott Heyward, BioIVT Inc. (Baltimore, MD), and human kidney and heart tissues were provided by Drs. Edward Kelly and Rheem Totah (University of Washington, Seattle, WA) (Basit et al., 2020a). All samples used in this study were de-identified and the sources of the samples were anonymous to us. No human research was conducted hence ethical review and informed consent were not required.

\section{Sample preparation}

i. Pediatric liver homogenate samples: The pediatric liver samples $(\sim 100 \mathrm{mg})$ were homogenized in $500 \mu \mathrm{L} 4 \%$ sodium dodecyl sulfate (SDS) and membrane solubilization buffer (1:1 ratio, v/v), containing $0.5 \%$ protease inhibitor cocktail (Thermo Fisher Scientific, Rockford, IL) with gentle mixing, as described previously (Prasad et al., 2014; Bhatt et al., 2019; Ahire et al., 2021). The samples were incubated for $60 \mathrm{~min}$ at $300 \mathrm{rpm}\left(15^{\circ} \mathrm{C}\right)$ to allow membrane protein solubilization and were then centrifuged at $16,000 \mathrm{x} \mathrm{g}$ for $15 \mathrm{~min}\left(4^{\circ} \mathrm{C}\right)$.

ii. Paired adult and pediatric liver and kidney samples: The liver and kidney samples ( $\quad 60$ mg) were homogenized with Mem-PER ${ }^{\mathrm{TM}}$ Plus Membrane Protein Extraction Kit (Thermo Fisher, Rockford, IL) using hand-held homogenizer followed by incubation for 30 min at 4 ${ }^{\circ} \mathrm{C}$ with gentle shaking (300 rpm). The homogenate was centrifuged at 16,000 xg for $15 \mathrm{~min}$, $4{ }^{\circ} \mathrm{C}$. After centrifugation, the non-membrane part was separated and the pellet containing 
membrane proteins was mixed gently in $4 \%$ SDS and the membrane solubilization buffer provided in the kit. The samples were incubated for $60 \mathrm{~min}$ at $300 \mathrm{rpm}\left(15^{\circ} \mathrm{C}\right)$ to allow membrane protein solubilization. The resultant samples were centrifuged at $16,000 \mathrm{xg}$ for 15 min at $4{ }^{\circ} \mathrm{C}$. The supernatant containing the total membrane fraction was used for the quantification of mARC1 and mARC2 protein (Prasad et al., 2016a). The average membrane protein recovery was 49 and $38 \mathrm{mg}$ membrane protein/g tissue of liver and kidney, respectively

iii. Pooled S9 fractions of liver, intestine, kidney, lung, and heart: Thirty to sixty mg of liver, intestine, kidney, lung, or heart tissue was weighed and homogenized in $500 \mathrm{~mL} 4 \%$ SDS: membrane solubilization buffer $(1: 1 \mathrm{v} / \mathrm{v})$ containing $0.5 \%$ protease inhibitor cocktail (Thermo Fisher Scientific, Rockford, IL) using a hand-held rotary homogenizer with plastic probes. All homogenized samples were centrifuged at $9000 \mathrm{x}$ g for $30 \mathrm{~min}$ at $4{ }^{\circ} \mathrm{C}$ and the supernatant (S9 fraction) was isolated using a protocol described previously (Bhatt et al., 2018).

iv. CHIM and human hepatocytes samples: In addition to the tissue samples, we also utilized previously processed (Zhang et al., 2020; Ahire et al., 2021) CHIM and hepatocytes samples.

The total protein concentration in the four sets of samples was determined by BCA assay (Pierce Biotechnology, Rockford, IL) and the samples were diluted to $1 \mathrm{mg} / \mathrm{mL}$ before trypsin digestion. The samples were stored at $-80{ }^{\circ} \mathrm{C}$ and thawed at $37^{\circ} \mathrm{C}$ before $\mu \mathrm{LC}-\mathrm{MS} / \mathrm{MS}$ analysis.

\section{Recombinant mARC1 and $\mathrm{mARC} 2$ protein calibration curve}

Recombinant mARC1 and mARC2 protein standards served as calibrators, which were serially diluted to prepare calibration curves ranging from 0.21 to $108 \mathrm{nM}$ and 0.14 to $74 \mathrm{nM}$ for mARC1 and $\mathrm{mARC} 2$, respectively. The total protein concentration for the diluted standard was adjusted 
to $1 \mathrm{mg} / \mathrm{mL}$ by adding human serum albumin (HSA) before trypsin digestion. The surrogate peptides of mARC1 (DLLPIK and VGDPVYLLGQ) and mARC2 (LSPLFGIYYSVE and WFTNFL) were assessed by LC-MS/MS. The peptides DLLPIK and LSPLFGIYYSVE were used as quantifier peptides (used for quantitative assessment) because of their higher mass sensitivity, and the remaining two were used as qualifier peptides (peptides used for the confirmation of peptide identity).

\section{Trypsin digestion and $\mu \mathrm{LC}-\mathrm{MS} / \mathrm{MS}$ analysis}

The samples were digested by trypsin using a previously described protocol (Ahire et al., 2021). Briefly, $80 \mu \mathrm{L}(1 \mathrm{mg} / \mathrm{mL}$ protein concentration) of the sample was mixed with $30 \mu \mathrm{L}$ of $\mathrm{ABC}$ buffer (100 mM, pH 7.8), $10 \mu \mathrm{L}$ of DTT $(250 \mathrm{mM})$, and $20 \mu \mathrm{L}$ of BSA $(0.02 \mathrm{mg} / \mathrm{mL})$, followed by denaturation for $10 \mathrm{~min}$ at $95^{\circ} \mathrm{C}$. The samples were cooled down to room temperature for 10 min before alkylation with $10 \mu \mathrm{L}$ of IAA $(500 \mathrm{mM})$ for $30 \mathrm{~min}$ in dark. The samples were subjected to protein precipitation by adding ice-cold acetone and keeping at $-80{ }^{\circ} \mathrm{C}$ for $1 \mathrm{hr}$. The protein pellets were recovered by centrifugation at $16,000 \mathrm{x} \mathrm{g}$ for $10 \mathrm{~min}$. The pellets were washed with $500 \mu \mathrm{L}$ ice-cold methanol, dried under vacuum for $30 \mathrm{~min}$, and then resuspended in $60 \mu \mathrm{L}$ of $\mathrm{ABC}$ buffer $(50 \mathrm{mM}, \mathrm{pH} 7.8)$. Trypsin digestion was then initiated by adding $20 \mu \mathrm{L}$ of trypsin (protein/trypsin ratio $\sim 80: 1$ ). The samples were digested for $16 \mathrm{~h}$ at $37{ }^{\circ} \mathrm{C}$ with gentle shaking $(300 \mathrm{rpm})$ before quenching by adding $5 \mu \mathrm{L}$ of $0.5 \%$ formic acid. The samples were stored in $-80{ }^{\circ} \mathrm{C}$ freezer before $\mu \mathrm{LC}-\mathrm{MS} / \mathrm{MS}$ analysis. A cocktail of mixture SIL peptides (internal standards), which contained $\sim 1 \mathrm{ng} / \mathrm{mL}$ mixture of four SIL peptides corresponding to each of the peptides being analyzed, was added to each sample. Trypsin digestion was performed in triplicate on three different days to evaluate the reproducibility of the results. 
The digested protein samples were analyzed using a Waters micro-flow LC system (M-class) coupled with Waters Xevo TQ-XS MS instrument supported by an ionKey interphase. The resultant peptides were separated on an iKey BEH C18 column $\left(130 \mathrm{~A}^{\circ}, 1.7 \mu \mathrm{m}, 150 \mu \mathrm{m} * 50\right.$ $\mathrm{mm})$ and nanoEase Symmetry C18 trap column (300 $\mu \mathrm{m} * 50 \mathrm{~mm})$ (Waters, Milford, MA). The detailed LC-MS/MS acquisition parameters are provided in Table S3.

\section{Data analysis}

LC-MS/MS data analysis was performed on open accessed Skyline (version 20.2) software (University of Washington, Seattle, WA) with a robust quantification strategy described previously (Bhatt and Prasad, 2018; Prasad et al., 2019). Briefly, the targeted peptide peaks were identified by matching the retention time and fragmentation patterns with the externally added stable isotope label (SIL) peptide cocktail. The analyte peptide peak area was normalized by the corresponding SIL peptide area. The externally added BSA served as an internal protein standard to address trypsin digestion variability. All experiments were performed in triplicate on three different days to check the reproducibility of the results. The coefficient of variation (CV) was measured across the triplicate analysis. Heat shock protein (CH60) was used as a mitochondrial marker, which was analyzed using a previously described method (Xu et al., 2018).

Kruskal- Wallis followed by Dunn's multiple comparison tests were used for the comparison of age-dependent protein abundance across different age groups. Mann-Whitney test was used for the analysis of mARC1 and mARC2 abundance between two groups (e.g., male and female). Paired t-test was applied to analyze the abundance in the paired kidney and liver samples. The pvalue $<0.05$ is considered significant.

\section{RESULTS}




\section{LC-MS/MS method for mARC quantification}

Chromatograms of surrogate peptides of mARC1 and mARC2 in representative human samples showed good signal-to-noise (S/N) ratios and specificity (Fig. 2A and 2B). Different product ions of individual peptides were aligned and the correlation between product ions was linear $\left(\mathrm{R}^{2}\right.$ value of >0.99). Between peptide correlation (quantifier versus qualifier peptides) also showed a strong correlation $\left(\mathrm{R}^{2}>0.98\right)$. Inter-day variability in peptide signals was minimal. The dynamic range of the method was linear between 6.74 to $54 \mathrm{nM}$ for $\mathrm{mARC} 1$ and 9.25 to $74 \mathrm{nM}$ for mARC2 (Fig. S1). Based on the signal-to-noise ratio criteria of 5:1, the lower limit of quantification (LOQ) was estimated to be $0.05 \mathrm{nM}$ and $4 \mathrm{nM}$ for mARC1 and mARC2, respectively. Other method validation parameters are listed in Table S4.

To test the sensitivity and linearity of the $\mu \mathrm{LC}-\mathrm{MS} / \mathrm{MS}$ method in human hepatocytes, mARC enzymes were quantified in 4000 to 1 million cells (24-6250 cells on-column; Fig. 2C). The abundance of mARC1 and mARC2 was linear up to cell count 1563 cell count on-column and showed saturation in the abundance likely due to saturation of the intensity or trypsin digestion.

\section{Tissue abundance of mARC enzymes}

mARC enzyme abundance was detected in i) pediatric liver homogenate samples, ii) paired pediatric and adult liver and kidney total membrane samples, and iii) pooled S9 fractions of liver, intestine, kidney, $\operatorname{lung}_{s}$, and heart. The same method was applied to CHIM cells to determine the relative abundance of mARC enzymes in different regions of the small intestine.

Pediatric human liver homogenate samples: mARC1 and mARC2 proteins were detected in all 79 tested pediatric liver homogenate samples, where the average hepatic abundance of mARC1 $(40.08 \pm 4.26 \mathrm{pmol} / \mathrm{mg}$ protein) was moderately but significantly higher $(\sim 1.5$-fold, $\mathrm{p}<0.05)$ than 
mARC2 $(24.58 \pm 4.02 \mathrm{pmol} / \mathrm{mg}$ protein) (Fig. 3A). The protein abundance was not associated with age (Fig. 3B-E) and sex (Fig. 3F-G) in the tested pediatric liver homogenate samples. Whereas, up to four-fold variability was observed within different pediatric age groups which could be due to other factors such as genetics, epigenetics, and environmental.

Paired pediatric and adult human liver and kidney total membrane samples: The average hepatic abundance of mARC1 ( 75 pmol/ mg membrane protein) and mARC2 ( 67 pmol/ mg membrane protein) was comparable in the paired pediatric and adult liver membrane fractions. The kidney mARC2 abundance $(\sim 120 \mathrm{pmol} / \mathrm{mg}$ membrane protein) in the paired individual samples was $\sim 9$-fold higher as compared to mARC1 ( 13 pmol/ mg membrane protein) (Fig. 4). mARC1 and mARC2 abundance in the paired liver and kidney membrane samples did not show inter-tissue correlation (Fig. S2).

The mitochondrial marker (CH60) showed <3-fold variability in differential tissue and paired samples (Fig. S3) indicating the presence of mitochondria at relatively consistent levels in S9 fractions.

Pooled S9 fractions of human liver, intestine, kidney, lung, and heart: mARC1 and mARC2 enzymes were also detected in the pooled S9 fractions of liver, kidney, intestine, and lung, however, mARC enzyme levels were below the LOQ in the heart $(\sim 0.6 \mathrm{pmol} / \mathrm{mg}$ protein $)$. The hepatic abundance of $\mathrm{mARC} 1$ in the $\mathrm{S} 9$ fractions was $38.60 \pm 1.60 \mathrm{pmol} / \mathrm{mg}$ protein, which was 5-, 9-, and 13- fold higher as compared to the kidney, intestine, and lung, respectively. Similarly, hepatic mARC2 abundance was $37.53 \pm 3.23 \mathrm{pmol} / \mathrm{mg}$ protein, which was 3 - and 16-fold higher as compared to the intestine and lung, respectively. mARC2 protein abundance in kidney S9 was 2.5-fold higher as compared to the liver S9 (Fig. 4C). Hepatic mARC1 and mARC2 protein levels were comparable in adult liver S9 samples. The average S9 protein recovery was 101, 38, 
59, 26, and $156 \mathrm{mg} \mathrm{S9}$ protein/g liver, intestine, kidney, heart, and lung, respectively. The scaled hepatic and kidney S9 abundance of mARC1 and mARC2 (pmol/gm of tissue) was comparable to the scaled hepatic and kidney membrane abundance (pmol/gm of tissue) (Table S5). mARC protein abundance in the CHIM cells: The exploratory analysis of CHIM samples obtained from our previous study (Zhang et al., 2020) revealed a greater than 2-fold difference in the expression of mARC1 in the upper part of the small intestine as compared to the ileum. Whereas mARC2 expression was not variable along the intestinal tract (Fig. S4).

\section{DISCUSSION}

The focus on the advancements of compounds with little to no oxidative metabolism using wellcharacterized high-throughput in vitro and in silico metabolism assays has lowered the contribution of CYP enzymes in drug metabolism over the past two decades (Cerny, 2016). Most often these chemotypes rely on different non-CYP enzymes for their oxidation, reduction, hydrolysis, and conjugation before renal excretion. mARC and $\mathrm{AO}$ are two such reductive enzyme families, which have been recently recognized to play important roles in the drug metabolism of newer chemical entities (Garattini and Terao, 2012; Sanoh et al., 2015; Rixen et al., 2019). For example, carbazeran and famciclovir are metabolized by AO, and ximelagatran and cimlanod are metabolized by mARC. However, a limited understanding of differential tissue expression and inter-individual variability (effect of age, sex, genetics, race, and disease mediated changes) of these enzymes is a critical knowledge gap, especially for mARC enzymes.

The mARC enzymes are recently discovered Mo-containing proteins that are involved in the myriad of the human physiological processes, e.g., lipid biosynthesis and NO homeostasis (Kotthaus et al., 2011; Klein et al., 2012), toxicity protection, e.g., metabolism of promutagenic N-hydroxy nucleobases and N-oxide metabolites (Krompholz et al., 2012), and in the 
metabolism of prodrugs and xenobiotics, e.g., melagatran and cimlanod (Clement and Lopian, 2003; Cowart et al., 2019b). For example, sulfamethoxazole (SMX) is oxidized to SMX hydroxylamine (SMX-HA) by cytochrome P450 2C9 (CYP2C9), which is further autoxidized to nitroso intermediate (a reactive moiety) leading to immunogenic reactions. The mARC enzyme system reduces SMX-HA to SMX and thus plays an important role in regulating hypersensitive reactions (Ott et al., 2014). Similarly, there are other examples where hydroxylamines containing drugs or active metabolites are associated with toxicity, e.g., nephrotoxicity of pentamidine (Lachaal and Venuto, 1989). Considering the emerging role of mARC enzymes in both endoand xeno-biotic metabolism, the understanding of the quantitative abundance of mARC enzymes across tissues, and their inter-individual variability (effect of age and sex) was warranted.

Here, we addressed this knowledge gap by quantifying and comparing the abundance of mARC enzymes in pediatric liver samples (age 0-18 yrs), paired adult liver and kidney, and five adult human tissues (liver, kidney, intestine, lung, and heart). The liver is the predominant organ that expresses both $\mathrm{mARC} 1$ and $\mathrm{mARC} 2$, but the abundance of the latter in the kidney (per mg S9 protein) was around 2- fold higher than in the liver. These data were confirmed by analysis of the paired membrane protein samples received from individual donors. In addition to the liver and kidney, both these enzymes are also expressed in the intestine and lung. These data signify that the mARC enzymes are important for normal cell function across organs. In fact knocking down murine mARC2 expression is associated with a substantial reduction in total body fat (Rixen et al., 2019). Moreover, caloric diet restriction in obese patients and metabolic disorders such as diabetes are associated with decreased abundance of mARC2 in the liver (Jakobs et al., 2014b; Neve et al., 2015). Although inhibitors or inducers of mARC enzymes are not characterized, it 
can be anticipated that perturbation of mARC activity by other drugs would lead to toxicity due to alteration of the physiological function of mARC enzymes.

Further, the quantitative information of mARC enzyme abundance across different human tissues is important in the understanding of the organ-specific drug disposition and development of whole-body adult PBPK models. For example, several mARC substrates have been developed to improve the oral absorption of amidine-containing drugs (e.g., ximelagatran and upomastat). The intestinal abundance data of mARC protein reported in this study will be useful in predicting intestinal activation of amidine-containing prodrugs. We also quantified mARC abundance across different age groups, and it was observed that mARC enzymes are equally important in both children and adults, and between males and females. Such a highly conserved abundance profile of mARC enzymes indicates their role in critical physiological processes. Indeed, mARC enzymes are involved in detoxification of N-hydroxylated nucleobases that are promutagenic and can lead to epigenetic adverse effects such as carcinogenicity (Krompholz et al., 2012; Plitzko et al., 2015).

The quantitative mARC expression data can be used in the prediction of metabolic clearance (CL) of mARC substrates. In particular, the intrinsic metabolic clearance via mARC enzymes may likely be extrapolated from the recombinant mARC enzymatic system to the tissue level assuming the recombinant system is fully active and the Michalis-Menton constant $\left(\mathrm{K}_{\mathrm{m}}\right)$ of mARC substrate is similar between both systems.

The tissue-specific milligram of $\mathrm{S} 9$ protein per gram of the tissue (MS9PPGT) of mARC enzymes across tissues should be considered (with the assumption of constant mitochondria recovery across the tissues) for the in vitro to in vivo extrapolation (IVIVE) in the optimum prediction of drug disposition. Usually, total clearance is considered in the scaling of drug 
metabolism to the entire organ level, however intracellular levels (pmol/mg or pmol/cell abundance) are important for the prediction of organ-specific toxicity. Thus, it is important to consider both total protein-normalized (pmol per mg) and per organ abundance (pmol per organ) data for the comparison of inter-tissue abundance. For example, hepatic mARC2 could be the primary enzyme involved in the metabolic clearance of mARC2 substrates due to higher $\%$ abundance in the liver, whereas a higher concentration of mARC2 (pmol/mg protein) in kidney will likely result in higher intracellular metabolite concentration.

There are some limitations of this study. We were able to detect mARC abundance in the pooled S9 fraction that is technically a post-mitochondrial fraction. Since mitochondria isolation generally requires $\sim 15,000 \mathrm{x}$ g centrifugation speed, the $9000 \mathrm{x}$ g centrifugation step used in the preparation of the S9 fraction is perhaps not sufficient to remove mitochondria completely. Detection of mitochondrial marker protein, CH60, confirmed the presence of mitochondria in S9 fractions. Levels of mitochondria remaining in the pooled S9 fractions were sufficient and consistent enough to enable a relative comparison of mARC levels across tissues. Also, the extrapolation of the tissue S9 data to the whole organ is based on two assumptions, i) uniform distribution of mARC enzymes across the tissues analyzed and ii) constant recovery of mitochondria during sample preparation across all the tissue. Further, we were unable to confirm the correlation between protein abundance and enzyme activity due to the lack of a probe substrate capable of distinguishing between the activities of mARC1 and mARC2.

In summary, we for the first time are reporting a comprehensive analysis of inter-individual in mARC enzyme abundance data including differential tissue abundance. The mARC abundance data could be integrated into the PBPK modeling of mARC substrates for the prediction of drugdrug interactions, tissue-specific toxicity, and pro-drug activation. 
DMD Fast Forward. Published on December 23, 2021 as DOI: 10.1124/dmd.121.000805

This article has not been copyedited and formatted. The final version may differ from this version. 
Authorship contribution

Participated in research design: Deepak Ahire, Lisa J. Christopher, Ramaswamy Iyer, Bhagwat

Prasad

Conducted experiments: Deepak Ahire, Abdul Basit

Contributed new reagents or analytic tools: Deepak Ahire, Abdul Basit

Performed data analysis: Deepak Ahire, Abdul Basit, Bhagwat Prasad

Wrote or contributed to the writing of the manuscript: Deepak Ahire, Lisa J. Christopher,

Ramaswamy Iyer, J. Steven Leeder, Bhagwat Prasad 


\section{References}

Ahire D, Basit A, Karasu M, and Prasad B (2021) Ultrasensitive quantification of drug-metabolizing enzymes and transporters in small sample volume by microflow LC-MS/MS. Journal of pharmaceutical sciences.

Basit A, Neradugomma NK, Wolford C, Fan PW, Murray B, Takahashi RH, Khojasteh SC, Smith BJ, Heyward S, and Totah RA (2020a) Characterization of differential tissue abundance of major non-CYP enzymes in human. Molecular Pharmaceutics 17:4114-4124.

Basit A, Neradugomma NK, Wolford C, Fan PW, Murray B, Takahashi RH, Khojasteh SC, Smith BJ, Heyward S, Totah RA, Kelly EJ, and Prasad B (2020b) Characterization of Differential Tissue Abundance of Major Non-CYP Enzymes in Human. Mol Pharm 17:4114-4124.

Bhatt DK, Basit A, Zhang H, Gaedigk A, Lee SB, Claw KG, Mehrotra A, Chaudhry AS, Pearce RE, Gaedigk R, Broeckel U, Thornton TA, Nickerson DA, Schuetz EG, Amory JK, Leeder JS, and Prasad B (2018) Hepatic Abundance and Activity of Androgen- and Drug-Metabolizing Enzyme UGT2B17 Are Associated with Genotype, Age, and Sex. Drug Metab Dispos 46:888-896.

Bhatt DK, Mehrotra A, Gaedigk A, Chapa R, Basit A, Zhang H, Choudhari P, Boberg M, Pearce RE, and Gaedigk R (2019) Age-and genotype-dependent variability in the protein abundance and activity of six major uridine diphosphate-glucuronosyltransferases in human liver. Clinical Pharmacology \& Therapeutics 105: 131-141.

Bhatt DK and Prasad B (2018) Critical issues and optimized practices in quantification of protein abundance level to determine interindividual variability in DMET proteins by LC-MS/MS proteomics. Clinical Pharmacology \& Therapeutics 103:619-630.

Cerny MA (2016) Prevalence of non-cytochrome P450-mediated metabolism in Food and Drug Administration-approved oral and intravenous drugs: 2006-2015. Drug Metabolism and Disposition 44:1246-1252.

Clement B and Lopian K (2003) Characterization of in vitro biotransformation of new, orally active, direct thrombin inhibitor ximelagatran, an amidoxime and ester prodrug. Drug metabolism and disposition 31:645-651.

Cowart D, Venuti RP, Lynch K, Guptill JT, Noveck RJ, and Foo SY (2019a) A Phase 1 Randomized Study of Single Intravenous Infusions of the Novel Nitroxyl Donor BMS-986231 in Healthy Volunteers. J Clin Pharmacol 59:717-730.

Cowart D, Venuti RP, Lynch K, Guptill JT, Noveck RJ, and Foo SY (2019b) A phase 1 randomized study of single intravenous infusions of the novel nitroxyl donor BMS-986231 in healthy volunteers. The Journal of Clinical Pharmacology 59:717-730.

Garattini E and Terao M (2012) The role of aldehyde oxidase in drug metabolism. Expert opinion on drug metabolism \& toxicology 8:487-503.

Havemeyer A, Lang J, and Clement B (2011) The fourth mammalian molybdenum enzyme mARC: current state of research. Drug metabolism reviews 43:524-539.

Jakobs HH, Froriep D, Havemeyer A, Mendel RR, Bittner F, and Clement B (2014a) The mitochondrial amidoxime reducing component (mARC): involvement in metabolic reduction of $\mathrm{N}$-oxides, oximes and N-hydroxyamidinohydrazones. ChemMedChem 9:2381-2387.

Jakobs HH, Mikula M, Havemeyer A, Strzalkowska A, Borowa-Chmielak M, Dzwonek A, Gajewska M, Hennig EE, Ostrowski J, and Clement B (2014b) The N-reductive system composed of mitochondrial amidoxime reducing component (mARC), cytochrome b5 (CYB5B) and cytochrome b5 reductase (CYB5R) is regulated by fasting and high fat diet in mice. PloS one 9:e105371.

Klein JM, Busch JD, Potting C, Baker MJ, Langer T, and Schwarz G (2012) The mitochondrial amidoxime-reducing component (mARC1) is a novel signal-anchored protein of the outer mitochondrial membrane. Journal of Biological Chemistry 287:42795-42803. 
Kotthaus J, Wahl B, Havemeyer A, Kotthaus J, Schade D, Garbe-Schönberg D, Mendel R, Bittner F, and Clement B (2011) Reduction of N $\omega$-hydroxy-l-arginine by the mitochondrial amidoxime reducing component (mARC). Biochemical Journal 433:383-391.

Krompholz N, Krischkowski C, Reichmann D, Garbe-Schönberg D, Mendel R-R, Bittner F, Clement B, and Havemeyer A (2012) The mitochondrial amidoxime reducing component (mARC) is involved in detoxification of N-hydroxylated base analogues. Chemical research in toxicology 25:2443-2450.

Lachaal M and Venuto RC (1989) Nephrotoxicity and hyperkalemia in patients with acquired immunodeficiency syndrome treated with pentamidine. The American journal of medicine 87:260-263.

Li CY, Hosey-Cojocari C, Basit A, Unadkat JD, Leeder JS, and Prasad B (2019) Optimized renal transporter quantification by using aquaporin 1 and aquaporin 2 as anatomical markers: application in characterizing the ontogeny of renal transporters and its correlation with hepatic transporters in paired human samples. The AAPS journal 21:1-8.

Llamas A, Chamizo-Ampudia A, Tejada-Jimenez M, Galvan A, and Fernandez E (2017) The molybdenum cofactor enzyme mARC: Moonlighting or promiscuous enzyme? Biofactors 43:486-494.

Maia LB and Moura JJ (2015) Nitrite reduction by molybdoenzymes: a new class of nitric oxide-forming nitrite reductases. JBIC Journal of Biological Inorganic Chemistry 20:403-433.

Neve EP, Köfeler H, Hendriks DF, Nordling $\AA$, Gogvadze V, Mkrtchian S, Näslund E, and IngelmanSundberg M (2015) Expression and function of mARC: roles in lipogenesis and metabolic activation of ximelagatran. PLoS One 10:e0138487.

Ott G, Plitzko B, Krischkowski C, Reichmann D, Bittner F, Mendel RR, Kunze T, Clement B, and Havemeyer A (2014) Reduction of sulfamethoxazole hydroxylamine (SMX-HA) by the mitochondrial amidoxime reducing component (mARC). Chemical research in toxicology 27:1687-1695.

Plitzko B, Havemeyer A, Kunze T, and Clement B (2015) The pivotal role of the mitochondrial amidoxime reducing component 2 in protecting human cells against apoptotic effects of the base analog N6-hydroxylaminopurine. Journal of Biological Chemistry 290:10126-10135.

Plitzko B, Ott G, Reichmann D, Henderson CJ, Wolf CR, Mendel R, Bittner F, Clement B, and Havemeyer A (2013) The involvement of mitochondrial amidoxime reducing components 1 and 2 and mitochondrial cytochrome b5 in N-reductive metabolism in human cells. Journal of Biological Chemistry 288:20228-20237.

Prasad B, Achour B, Artursson P, Hop CE, Lai Y, Smith PC, Barber J, Wisniewski JR, Spellman D, and Uchida Y (2019) Towards a consensus on applying quantitative LC-MS/MS Proteomics in translational pharmacology research: a White Paper. Clinical pharmacology and therapeutics 106:525.

Prasad B, Evers R, Gupta A, Hop CE, Salphati L, Shukla S, Ambudkar SV, and Unadkat JD (2014) Interindividual variability in hepatic organic anion-transporting polypeptides and P-glycoprotein (ABCB1) protein expression: quantification by liquid chromatography tandem mass spectroscopy and influence of genotype, age, and sex. Drug Metab Dispos 42:78-88.

Prasad B, Gaedigk A, Vrana M, Gaedigk R, Leeder JS, Salphati L, Chu X, Xiao G, Hop CE, and Evers R (2016a) Ontogeny of hepatic drug transporters as quantified by LC-MS/MS proteomics. Clinical Pharmacology \& Therapeutics 100:362-370.

Rixen S, Havemeyer A, Tyl-Bielicka A, Pysniak K, Gajewska M, Kulecka M, Ostrowski J, Mikula M, and Clement B (2019) Mitochondrial amidoxime-reducing component 2 (MARC2) has a significant role in N-reductive activity and energy metabolism. Journal of Biological Chemistry 294:17593-17602.

Sanoh S, Tayama Y, Sugihara K, Kitamura S, and Ohta S (2015) Significance of aldehyde oxidase during drug development: effects on drug metabolism, pharmacokinetics, toxicity, and efficacy. Drug metabolism and pharmacokinetics 30:52-63. 
Schneider J, Girreser U, Havemeyer A, Bittner F, and Clement B (2018) Detoxification of trimethylamine $\mathrm{N}$-oxide by the mitochondrial amidoxime reducing component mARC. Chemical research in toxicology 31:447-453.

Schwarz G, Mendel RR, and Ribbe MW (2009) Molybdenum cofactors, enzymes and pathways. Nature 460:839-847.

Xu M, Saxena N, Vrana M, Zhang H, Kumar V, Billington S, Khojasteh C, Heyward S, Unadkat JD, and Prasad B (2018) Targeted LC-MS/MS proteomics-based strategy to characterize in vitro models used in drug metabolism and transport studies. Analytical chemistry 90:11873-11882.

Zhang H, Wolford C, Basit A, Li AP, Fan PW, Murray BP, Takahashi RH, Khojasteh SC, Smith BJ, and Thummel KE (2020) Regional proteomic quantification of clinically relevant non-cytochrome P450 enzymes along the human small intestine. Drug Metabolism and Disposition 48:528-536. 


\section{Financial support}

This work was supported by Eunice Kennedy Shriver National Institute of Child Health and Human Development (NICHD), NIH grant\# [R01.HD081299]. LC-MS/MS analysis of mARC proteins was funded by Bristol Myers Squibb (BMS). The NICHD Brain and Tissue Bank for Developmental Disorders at the University of Maryland is funded by the NIH contract, [N01HD-9- 0011/HHSN275200900011C].

\section{Conflict of Interest}

Deepak Ahire, Abdul Basit, J. Steven Leeder, and Bhagwat Prasad have declared no conflict of interest. Lisa J. Christopher and Ramaswamy Iyer are employees and stockholders of Bristol Myers Squibb. 


\section{Figure Legends}

Fig. 1. Reduction mechanism of N-oxygenated compounds by mARC enzyme system (A) and examples of $\mathrm{N}$-oxygenated endobiotics and xenobiotics metabolized by mARC enzymes (B).

Fig. 2: Representative chromatograms of mARC1 (A) and mARC2 (B). Top panels: surrogate peptides (light peptides); Bottom panels: - SIL peptide (IS) used for protein quantification in digested human liver homogenate. Application of developed ultrasensitive $\mu$ LC-MS/MS method for the quantification of mARC1 and mARC2 abundance in low on-column hepatocytes counts (C and D, respectively). Both mARC1 and mARC2 were detectable at a level of 24 hepatocytes on-column and the response were linear up to 3125 hepatocytes on-column. The inset in each chromatogram shows the MS/MS fragment type (e.g., y3, y4, y5), m/z rations, and ionization state $(+v e)$.

Fig. 3: Box and whisker plots of absolute abundance of mARC1 and mARC2 enzymes in pediatric liver homogenates $(n=79)(A)$. Age-dependent association of mARC1 and mARC2 in pediatric liver samples $(n=79)$ on continuous (B and C) and categorical (D and E) scales. Association of sex-with mARC1 and mARC2 abundance (F and G).

Fig. 4: Differential tissue abundance of mARC1 and mARC2 in individual paired adult liver and kidney membrane fractions $(n=15)(A$ and $B)$ and in pooled $S 9$ fractions of human liver $(n=50)$, intestine $(n=20)$, kidney $(n=22)$, and lung $(n=11)(C$; data presented as mean and SD of the technical replicates of the pooled sample), and. ** paired t-test, $\mathrm{p}$ value $<0.0001$. 
Fig. 1.

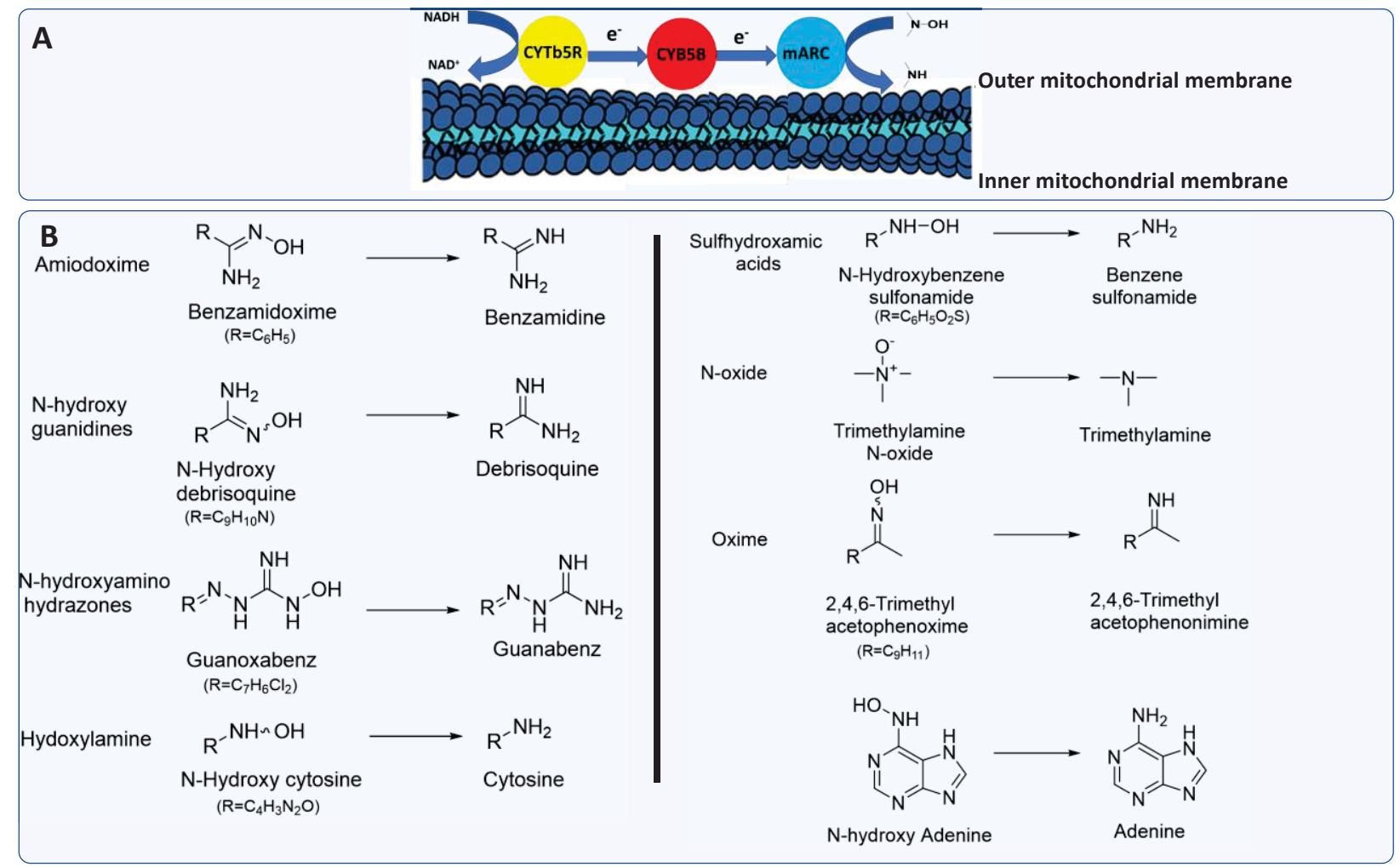


Fig. 2.
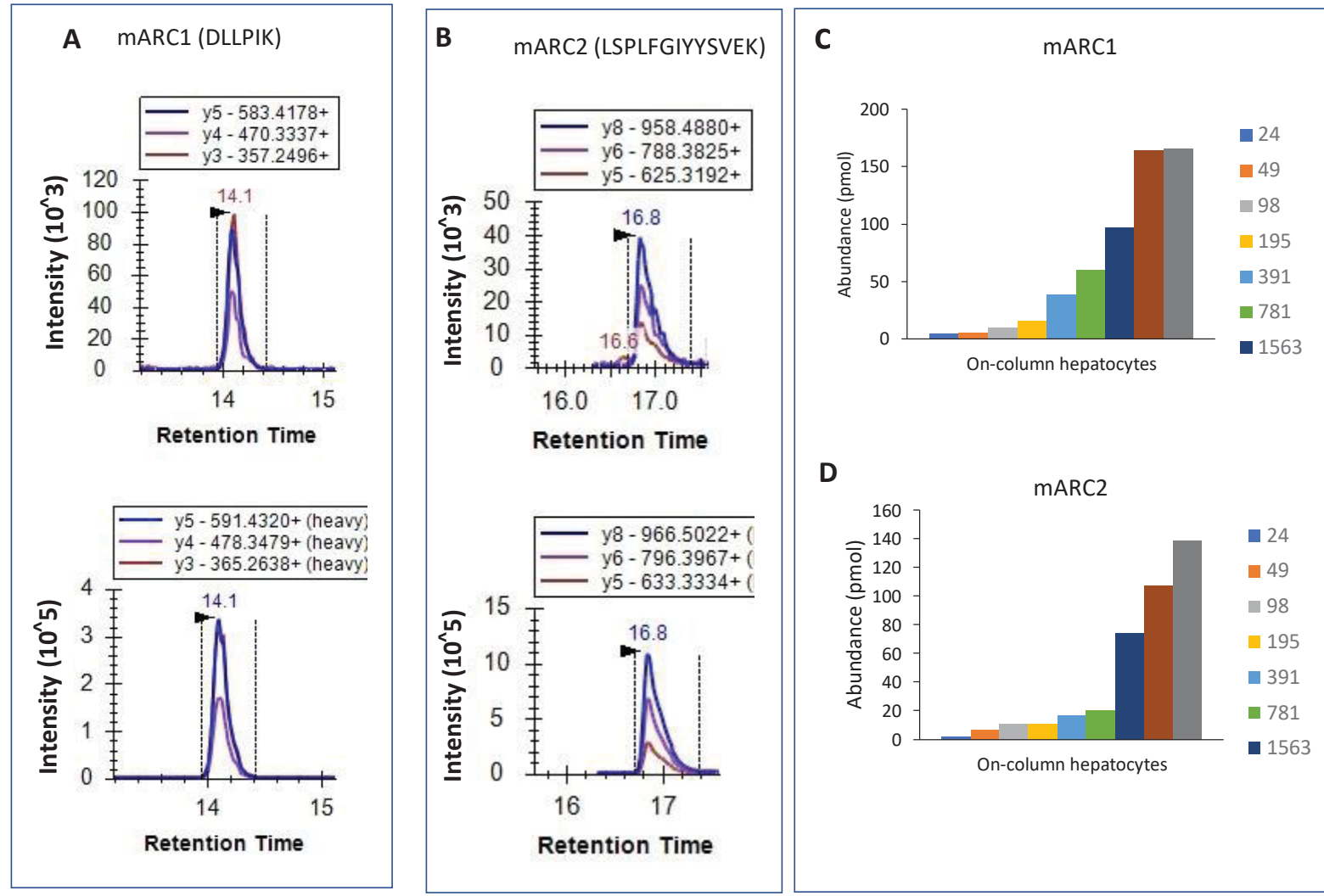
Fig. 3.
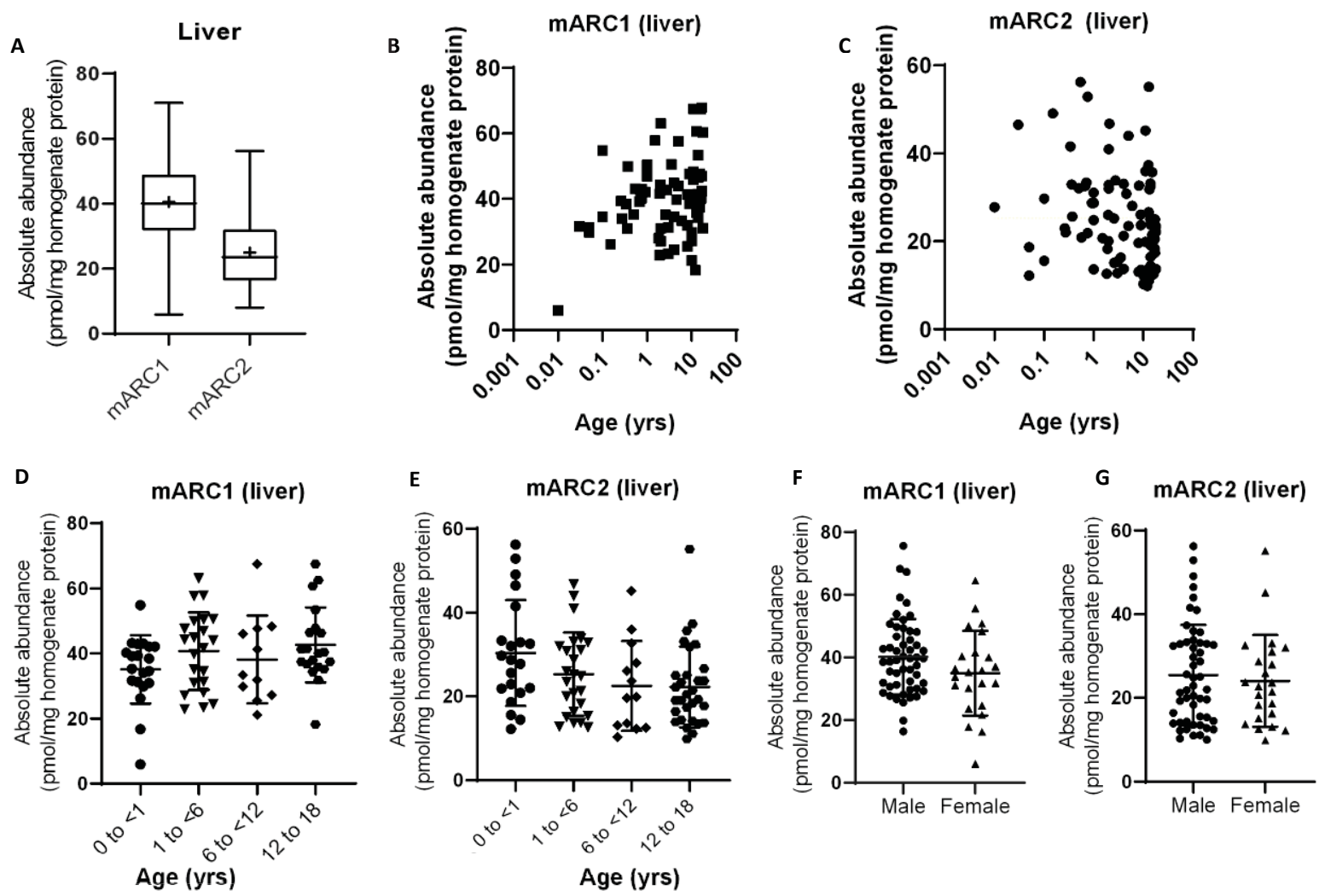
DMD Fast Forward. Published on December 23, 2021 as DOI: 10.1124/dmd.121.000805 This article has not been copyedited and formatted. The final version may differ from this version.

Fig. 4.
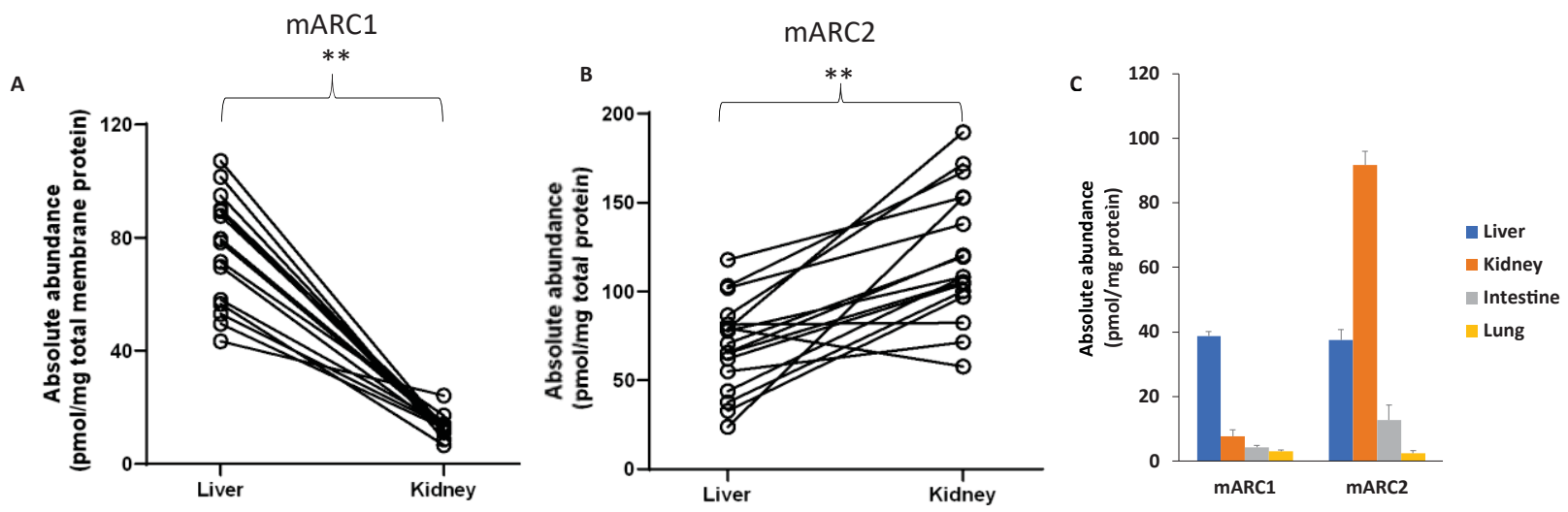

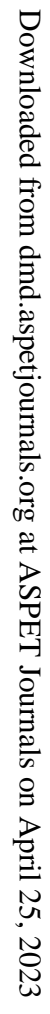

